\title{
Materials for nuclear industry: some historical perspectives
}

\author{
P R VASUDEVA RAO® \\ Homi Bhabha National Institute, Mumbai 400 094, Maharashtra, India \\ E-mail: vasudeva@hbni.ac.in
}

MS received 29 March 2019; revised 17 May 2019; accepted 22 May 2019; published online 14 October 2019

\begin{abstract}
Materials play a key role in the safe and economical operation of nuclear reactors. Materials used in reactors also have to meet stringent chemical specifications for efficient performance. Commercial scale realization of nuclear materials has been a challenge to the nuclear industry. The history of development of nuclear materials has fascinating and unique examples of theoretical prowess as well as innovative experimentation and success in nuclear material development is characterized by synergy between the domains of laboratory research and industry. This paper describes the development of some of the important nuclear materials (uranium, plutonium, zirconium, boron, sodium and graphite), providing a historical perspective.
\end{abstract}

Keywords. Nuclear materials; uranium; plutonium; zirconium; boron; graphite; sodium; lanthanides; history; scale-up.

\section{Introduction}

Among the 118 elements known today, some elements and their compounds play important roles in nuclear energy, such as fuel, neutron absorber, moderator, structural material, coolant, etc. For example, uranium and plutonium are used as nuclear fuel; zirconium is used as the clad material for the fuel as well as material for structural components; boron is used (as carbide) as a control rod material; sodium in liquid form is used as a coolant in fast reactors. Many of the elements associated with nuclear energy have unique properties and history. The development of nuclear materials on commercial scale has been an important and decisive element of the programs for nuclear energy.

Nuclear energy development was indeed a mother of several complex materials technologies. The Manhattan Project during World War II provided the impetus for development of high purity ("Nuclear Grade") materials on commercial scale. The emphasis on "high purity for high performance" has continued to provide developmental challenges for nuclear scientists. Materials used in nuclear reactors should not only have physical and chemical properties that enable them to perform in the hostile conditions inside the reactor core; materials playing

*For correspondence roles other than as fuels or control rod materials should also have very low neutron absorption so that they do not affect neutron economy. Fuel materials should also have very low concentrations of impurities that can cause parasitic neutron absorption (e.g., lanthanides, particularly $\mathrm{Eu}$ and $\mathrm{Gd}$; $\mathrm{Cd}$; boron). Techniques of analysis developed as an offshoot of nuclear science, e.g., neutron activation analysis, have enhanced the capability to determine a host of impurities in materials at trace levels in a simultaneous and non-destructive manner. Several elements (particularly lanthanides and actinides) have posed great challenges in isolation and purification. The development of separations schemes for such elements has significantly contributed to the maturity and expansion of separations sciences.

The history of nuclear materials also has examples of fascinating initiatives and innovative R\&D efforts that led to scaling up of the production of the materials. Early involvement of industry in the productionrelated developmental programs has also paid rich dividends in such instances.

This article explores the production of some of the materials such as uranium, plutonium, graphite and zirconium from a historical perspective. This paper also presents some examples of nuclear material development, highlighting the innovation and multidisciplinary team efforts. 


\section{Uranium}

As early as 1805 , uranium had been purified by ether extraction by Bucholz. ${ }^{1}$ Ether extraction was also used by Frederick Soddy in 1905 to prepare kg quantities of pure uranium in the course of studies on radioactive decay. ${ }^{2}$ All these studies were performed at laboratory scale, and at the time of Manhattan Project, no industrial scale production existed. The cost of uranium in the open market in USA at that time was around $\$ 1000$ per pound. ${ }^{3}$ During the early efforts to set up "piles" in Columbia by Fermi's team, it was quickly inferred that the uranium and graphite used needed to be of high purity. The first uranium (oxide) for the piles came from Eldorado Gold Mines Ltd., of Canada, essentially the residue left after removal of radium. However, this uranium was not adequately pure. "Fermi asked some of the Columbia chemists to analyse it, and the list of impurities was so long that it looked like a fair sample of the periodic system", according to Samuel Allison, Director of Metallurgical Laboratory under Manhattan Project. ${ }^{4}$ To expedite the production of pure uranium on a large scale, Edward Mallinckrodt, Jr., the president of Mallinckrodt Chemical Works, was approached by Arthur Compton, the Nobel prize winning physicist and the Director of the Manhattan District's Plutonium Research Project at the Metallurgical Laboratory at the University of Chicago, in April 1942 with a request to help USA in processing uranium for the Manhattan project. The plant, deploying the ether extraction process, was set up in about three months, and Mallinckrodt supplied nearly all uranium required for the Chicago pile.

"Delivery (of the pure oxide) started in July 1942 at a rate of 30 tons a month...it was a remarkable achievement to have developed and put into production on a scale of the order of one ton per day a process transforming grossly impure commercial oxide to oxide of a degree of purity seldom achieved even on a laboratory scale.". 5 By 1957, Mallinckrodt had produced over 50,000 tonnes of uranium oxide at its plant at St. Louis.

Parallel to the efforts of Mallinckrodt, Frank Spedding at Iowa State College also established a uranium production facility. The group led by Harley Wilhelm developed a method for reduction of uranium tetrafluoride to uranium metal and casting of uranium metal, making it possible to cast large ingots at lower cost. This process came to be known as Ames process and became the accepted route for the production of uranium metal. In the month of November 1942, the process was scaled up, and two tonnes of high-purity uranium metal were sent to Chicago. Between 1942 and 1945, over 1000 tonnes of uranium metal were produced at Ames laboratory in a scaled-up plant set up in a wooden house which had served earlier as a women's gymnasium! ${ }^{6}$

\section{Graphite}

The story of graphite in nuclear energy begins with a letter from Leo Szilard to Enrico Fermi, written on 3rd July 1939: "Dear Fermi, this is to keep you informed of the trend of my ideas concerning chain reactions. It seems to me now that there is a good chance that carbon might be an excellent element to use in place of hydrogen..". 7 Similar expectation that graphite could serve as a good moderator was also among other researchers, including Germans. Fission was discovered in Germany, and thus Germany had a natural lead in development of nuclear energy. However, Walther Bothe, student of Max Planck (and subsequently Nobel laureate for his development of the coincidence counting technique), measured the absorption cross section of carbon, and obtained a value of $6.4 \times 10^{-27} \mathrm{~cm}^{2}$-over twice the value obtained by Fermi, and concluded that graphite is not a good moderator; Von Halban and Kowarski at Cambridge also overestimated the cross section. It is very likely that both these groups had used impure graphite. ${ }^{8}$ However, Szilard's incisive approach and Fermi's rigour helped them to recognise the issue and resolve the same. Perhaps this is one unique aspect that helped the American groups to move ahead of Germans in achieving a nuclear chain reaction.

The "piles" set up in Columbia constituted the first large-scale use of graphite in nuclear industry. "[After the Einstein letter] ... help came along to the tune of $\$ 6,000$ a few months after and the $\$ 6,000$ were used in order to buy what seemed at that time when the eye of physicists had not yet been distorted-a huge amount of graphite... So, physicists on the seventh floor of Pupin Laboratories started looking like coal miners, and the wives to whom these physicists came back tired at night were wondering what was happening. Well, what was happening was that in those days we were trying to learn something about the absorption properties of graphite, because perhaps graphite was no good. So, we built columns of graphite, maybe four feet on the side or something like that, may be 10 feet high. It was the first time when apparatus in physics, and these graphite columns were apparatus, was so big that you could climb on top of it-and you had to climb on top of it".

As regards "nuclear grade" graphite, "it was not in the lab but over lunch with Fermi and two men from National Carbon Company (MacPherson and 
Hamister) that Szilard made one decisive discovery. $\mathrm{He}$ pushed his guests for more details about the impurities in commercial grade graphite, as one by one, he named elements that might absorb neutrons. Then, jokingly, he asked "You wouldn't put boron into your graphite, would you?" The two men looked at each other in embarrassed silence.. "As a matter of fact", one said, "samples of graphite that come from one of our factories contain boron, because we manufacture in that factory, also graphite electrodes for electric arcs, into which boron is customarily put". ${ }^{10}$ If this question had not been asked, it is quite possible that U.S researchers would have rejected graphite as a moderator. This discussion led to the development and commercial scale production of "Nuclear Graphite".

The first graphite employed in the piles had 1-2 ppm boron. This graphite (AGX grade-electrode grade) was produced by National Carbon Company and US Graphite Company. In early 1942, somewhat purer carbon was produced by Speer carbon company, and this gave a pile with $k>1.0$ for the first time. Hammister (senior scientist at research labs of National Carbon Company) and MacPherson, his associates, took up the challenge. They increased graphitization temperature to $2800 \mathrm{deg} \mathrm{C}$, which reduced impurity levels. Atcheson Graphite Ordinary Temperature (AGOT) grade graphite with $0.5 \mathrm{ppm}$ boron was used for the first time in Oak Ridge X-10 reactor. The historical review on Nuclear graphite by Eatherly ${ }^{11}$ chronicles the development of pure graphite production on large scale for the nuclear industry.

\section{Plutonium}

Plutonium has a unique place in science history. It is the first man-made element to be produced in "visible" amounts; the only man-made element to be produced in tonne scale, and one of the most dangerous poisons to be handled by man in large quantities. Most unique, however, is the fact that it has a fascinating history: it was discovered and studied under most difficult conditions of secrecy and urgency.

Plutonium was discovered on December 14, 1940, and the first isotope to be discovered was $\mathrm{Pu}-238$. The chemical identification of plutonium as a new element by A.C. Wahl took place in the night of February 23, $1941 .{ }^{12}$ Plutonium was prepared in $\mathrm{mg}$ quantities in November 1943, gram quantities in March 1944 and kilogram quantities in mid-1945. Thus, production of plutonium was scaled up from atoms to kilograms in a matter of a few years!
The efforts that were behind the separation of pure plutonium for the first time, in microgram quantities, are best described in the words of Glenn T. Seaborg himself: (1.2 kg of uranium was irradiated with neutrons for about a week to produce a microgram of $\mathrm{Pu}$ ). "On a Monday morning in March, Segre and I carried our uranium sample up two flights of stairs in the chemistry building.. We dissolved the target in 21 of ether and poured the mixture into the extraction apparatus, where most of the uranium compound dropped out, leaving the element 93, any 94 that was formed and fission products in the solution. We heated this to reduce the volume.. we added carriers that would combine with the element 93.. we poured this suspension into a centrifuge tube.. carried it to Crocker laboratory (that had a centrifuge).. we spun the sample in the centrifuge... (After centrifugation) walked back to our work room.. dissolved it in acid, oxidized it, and added carriers again.. Then it was back to the Crocker Laboratory, then back to our room.. we called it a night at 10 p.m., but were back in the morning to continue the process, six cycles of reprecipitation and centrifugation over the next three days (to produce the pure Pu sample of less than 1 microgram)..". ${ }^{13}$

Speaking about the challenge of devising the separation scheme, Seaborg says "The chemistry group's challenge was to come up with a process by which we could separate out the plutonium from all the material in the aftermath of the chain reaction. The process would have to work on a large scale. The plutonium would be present in a concentration of about 250 parts per million. That meant that there would be about a half a pound of plutonium in each ton of irradiated uranium. The uranium would also contain a large selection of intensely radioactive fission products. So, our challenge was to find a way to separate relatively small amounts of plutonium from tons of material so intensely radioactive that no one could come near...We would have to develop this process for an element that now (in 1942) existed in such minute amounts that no one had ever seen it. All our knowledge of it was based on the secondary evidence of tracer chemistry-measurements of radioactivity and deduced reactions". ${ }^{14}$

Considering the urgency in scaling up the production of plutonium, Seaborg devised a large number of separation schemes. The research was organised in such a way as not to miss any bets. Although it became evident that a conservative precipitation process would become the method of choice for the large scale production of plutonium, other possibilities were not ignored. "In Nov. 1943, Seaborg listed 17 possible approaches to plutonium extraction and decontamination". ${ }^{15}$ The basic flowsheet of the Bismuth 
Phosphate process for the separation of plutonium from irradiated uranium target at Hanford was developed based on studies carried out at mg level. Thus, the scale-up between the ultramicrochemical experiments to the final Hanford plant amounts to a factor of about $10^{9}$, surely the greatest scale-up factor ever attempted. ${ }^{16}$ However, the development was so robust that at the Hanford plant, from the very beginning, decontamination factors were better than anticipated and reached the overall value of $10^{8} .{ }^{17}$

\section{Lanthanides}

Lanthanides constitute an important group of fission products. Compounds of some of the lanthanides such as europium and gadolinium are also deployed in nuclear reactors as neutron absorbers. Some of the lanthanide isotopes are useful indicators for the "burnup" reached by the nuclear fuel. Lanthanides also have a wide variety of other applications. Many applications of lanthanides require availability of the material in high purity. Before the advent of nuclear energy, production of lanthanides was generally limited to small scale. It is not an exaggeration to say that the advent of nuclear energy led to the large scale production of lanthanides, even though it is known that as early as the early part of twentieth century, pioneers like Charles James did produce large quantities of some of the lanthanides. ${ }^{18}$

Lanthanides have always been a challenge to the separation chemist because of the closeness in chemical properties. For example, Paul Emile Lecoq de Boisbaudran who discovered dysprosium as an impurity in erbium oxide, tried repeated precipitation over 30 times, but was unable to obtain pure dysprosium from erbium; he gave the element the name Dysprosium, meaning "difficult to get"! ${ }^{19}$ Similarly, thulium needed a large number of steps of bromate fractional recrystallisation to establish homogeneity and purity. In his paper, Charles James mentions that the spectrum of thulium showed no change after about 15000 steps of recrystallisation. ${ }^{20}$

The separation of lanthanides was studied in depth as part of Manhattan Project. In fact, the lanthanide Promethium was discovered during the work on Manhattan project. ${ }^{21}$ Harold Spedding at Iowa State College, led a group of chemists that developed the bulk scale separation of lanthanides by ion exchange process. Between 1944 and 1945, almost $100 \mathrm{~kg}$ of pure cerium metal was produced, to make cerium sulphide crucibles for the plutonium program. The first reports on ion exchange chromatography for separation of lanthanides appeared in 1947, after the declassification of the work on lanthanides during the Manhattan project. In fact, one can find nine classic papers on ion exchange chromatography in one issue of Journal of American Chemical Society (Volume 69).

\section{Zirconium}

Production of zirconium metal in powder form was demonstrated as early as 1824 by Berzelius. ${ }^{22}$ The first practical method for producing zirconium metal of reasonably good purity was reported by Van Arkel, DeBoer and Fast in $1925 .^{23}$ However, only a few hundred pounds of zirconium were produced in the United States in 1945, and it costs more than \$300/ $1 \mathrm{~b}{ }^{24}$ The acclaimed Kroll's process for zirconium metal production was reported in $1946 .{ }^{25}$

The realization that the presence of hafnium in naturally occurring zirconium was responsible for neutron absorption was an important development that led to the use of zirconium in nuclear reactors. Albert Kaufman of MIT suggested that zirconium might be useful for use as cladding if neutron absorption could be reduced. Herbert Pomerance, an experimental spectroscopist in Oak Ridge discovered that the neutron absorption in zirconium was in fact caused by hafnium. Herbert Pomerance used an innovative Maytag-powered pile oscillator developed by Woolan at ORNL to make the measurements on the hafnium content of zirconium. ${ }^{26}$ It was soon established that purified zirconium metal has an acceptable neutron absorption cross section due to reduction in the hafnium content. In 1947, Fischer and Chalybaeus reported a method for the separation of hafnium from zirconium by solvent extraction using methyl isobutyl ketone as solvent. ${ }^{27}$ The industrial scale development of zirconium production was due to the decision taken by Admiral Rickover of US Navy to use zirconium as structural material for the submarine (Nautilus) reactor. After the decision of Rickover, there was a great interest in developing a process that could produce a large quantity of zirconium at a much lower cost. By 1953, hafnium-free zirconium oxide could be produced at a price of well under $\$ 5 / \mathrm{lb}^{28} \mathrm{~A}$ process based on solvent extraction with tri-n-butyl phosphate was reported for the separation of hafnium from zirconium in $1958 .{ }^{29}$ Between 1957 and 1963, over ten million pounds of zirconium and about a quarter of million pounds of hafnium were delivered for utilisation in naval reactors, at an average cost of $\$ 6 / 1 \mathrm{~b} .{ }^{30}$ 
Zirconium is a unique case of nuclear material, where high purity did not help; instead, alloying provided the breakthroughs! The first zirconium alloy (Zircaloy-I) was found to have increasing rate of corrosion over time, so it was not deployed. A number of new alloy compositions were studied, and one had a small amount of stainless steel added accidentally, and this showed better behavior. Based on this observation, Rickover decided to establish production of an alloy subsequently called as Zircaloy-2-before an ingot had ever been melted, fabricated or tested for corrosion.

\section{Boron}

Back home, the development of enriched boron carbide for the Indian fast reactor program is an excellent example of the impact of synergistic efforts between R\&D and industrial units of the Department of Atomic Energy. Boron carbide has been the material of international choice as a material for use as a control rod in nuclear reactors. Boron carbide used in control rods of fast reactors has to be enriched in B-10 isotope because of the lower neutron absorption cross section at high neutron energies. Boron has two isotopes B-10 and $\mathrm{B}-11$; the B-10 content in natural boron is 20 at.\%. The boron used to make boron carbide for small fast reactors like the Fast Breeder Test Reactor at Kalpakkam has to have a high level of enrichment (90 at.\% B-10 used in FBTR). For large reactors like the 500 MWe Prototype Fast Breeder Reactor, the enrichment needed can be lower (e.g., 65 at.\% used for PFBR). The enrichment of boron by ion exchange process was developed by Bhabha Atomic Research Centre (BARC) and further improved to a pilot plant scale at India Gandhi Centre for Atomic Research (IGCAR). The distillation process for enrichment was developed by the Heavy Water Board. The conversion of boric acid to elemental boron through precipitation as $\mathrm{KBF}_{4}$, and the electrowinning of elemental boron from chloride-flouride salt medium at high temperature $\left(800{ }^{\circ} \mathrm{C}\right)$ were demonstrated at IGCAR based on the technology developed at BARC, and the conversion of boron to boron carbide and the consolidation of boron carbide as pellets were demonstrated at BARC. The close interaction between BARC, IGCAR and HWB and the involvement of the HWB through the developmental steps were responsible for the successful indigenous production of enriched boron carbide required for PFBR.

\section{Sodium}

Liquid sodium is a material with physical properties that make it attractive for use as a coolant for nuclear reactors and sodium is a preferred coolant for fast reactors. It has high thermal conductivity, low melting point $(371 \mathrm{~K})$ and high boiling point $(1156 \mathrm{~K})$. Consequently, it has low vapor pressures at reactor operating temperatures. Its low density $(0.9 \mathrm{~g} / \mathrm{cc})$ and low viscosity are other physical properties that are interesting from the engineering viewpoint. However, due to its high chemical reactivity, producing high purity and maintaining the purity during reactor operation are challenges. Even though pure sodium is benign to stainless steel or ferritic steel, the structural materials used in fast reactors, the non-metallic impuritiesoxygen and carbon-present in sodium can have great impact on its use. The corrosion of structural steels in fact, depends on the oxygen concentration in sodium and the limit of oxygen impurity in reactor grade sodium is thus set to $10 \mathrm{ppm}$ or less. Carburisation is detrimental to structural integrity of steels, and carbon can be transported from one part of sodium circuit to another in fast reactors, depleting carbon in some part and causing carburization in another part.

Commercial, pool type Fast Reactors typically have over 1000 tonnes of liquid sodium. Thus, producing pure sodium on large scale is a technology that has to be mastered for indigenous development of fast reactors.

The EBR-I reactor, built at Idaho, USA was the first reactor (a fast reactor) to use liquid alkali metal coolant (sodium-potassium alloy). It achieved criticality in August 1951, and was the first to produce nuclear electricity. The first Indian fast reactor - Fast Breeder Test Reactor at the Indira Gandhi Centre for Atomic Research (IGCAR) at Kalpakkam-uses around 100 tonnes of sodium as its coolant. In the very early stages of the fast reactor program, the analytical techniques required for the characterization of sodium were standardized at BARC and subsequently at IGCAR. Sodium required for FBTR was supplied by $\mathrm{M} / \mathrm{s}$ Alkali Metals Limited, a private manufacturer at Hyderabad, India, but needed to be purified to meet the reactor requirements. A purification loop was set up and the entire sodium (over a hundred tonnes) to be loaded in FBTR was purified at IGCAR.

\section{Conclusions}

The history of the development of nuclear materials has shown that a knowledge base on the material requirements and the role of impurities, and the 
availability of industrial support in large scale production are a key to the success of the development program. One also sees that a scale up in a short time scale is possible if the industry is involved at an early stage of development. Finally, the innovative steps taken by the teams engaged in the efforts related to nuclear materials are indeed inspiring and deserve study by young researchers.

\section{References}

1. Bucholz C F 1805 Neues allgem J. Der Chem. 4157

2. Soddy F and Mackenzie T D 1907 The relation between uranium and radium Philos. Mag. 14272

3. Compton A 1956 Atomic Quest-A Personal Narrative (Oxford: Oxford University Press) p. 91

4. Allison S K 1962 Initiation of chain reaction-the search for pure materials IAEA Bull. 4-0 12

5. Smyth H D 1945 Atomic Energy for Military Purposes, the Official Report on the Development of Atomic Bomb under the Auspices of the United States Government, 1940-1945 (New York: Maple Press)

6. Payne C S 1992 The Ames Project: Administering Classified Research as a Part of the Manhattan Project at Iowa State College, 1942-1945 (Iowa State University dissertation) p. 72

7. Lanouette W and Silard B 1992 Genius in the Shadows-A Biography of Leo Szilard (London: Macmillan Publishing Co.) p. 195

8. Rhodes R 1986 The Making of the Atomic Bomb (New York: Simon and Schuster Paperbacks) p. 344

9. Fermi E 1955 Physics at Columbia University-the genesis of the nuclear energy project Phys. Today $\mathbf{8} 12$

10. Ref.7, p. 222

11. Eatherly W P 1981 Nuclear graphite: the first years $J$. Nucl. Mater. 10055

12. Seaborg G T 1976 Early History of Heavy Isotope Research at Berkeley Report no. Pub-97 (Lawrence Berkeley Laboratory) p. 28
13. Seaborg G T and Seaborg E 2001 Adventures in the Atomic Age: From Watts to Washington (Farrar, Straus and Giroux, New York) p. 75

14. Ref.13, p. 96

15. Katz J J 1990 Remembering the early days of the Met Lab in CONF-9010297-1, Proceedings of the Welch Foundation Conference on Chemical Research, October 22-23, 1990, (USA) p. 7

16. Hoffman D C, Ghiorso A and Seaborg G T 2000 The Transuranium People-The Inside Story (London: Imperial College Press) p. 71

17. Ref. 16, p. 74.

18. Separation of Rare Earth Elements by Charles James. National Historic Chemical Landmark, Dedicated October 29, 1999. University of NewHampshire, Durham, NH. http://www.acs.org/content/acs/en/education/ whatischemistry/landmarks/earthelements.html

19. C H Evans (Ed.) 1996 Episodes from the History of the Rare Earth Elements (Dordrecht: Kluwer Academic Publishers) p. 62

20. James C 1911 J. Am. Chem. Soc. 331332

21. Marinsky J A, Glendenin L E and Coryell C D 1947 The chemical identification of radioisotopes of neodymium and element $61 \mathrm{~J}$. Am. Chem. Soc. 692781

22. Berzelius J 1825 Poggendorff Ann. 4121

23. Van Arkel A E 1925 Z. Anorg. Chem. 148345

24. Rickover H G 1975 in History of the Development of Zirconium Alloys for Use in Nuclear Reactors $\mathrm{H} G$ Rickover, L D Geiger and B Lustman (Eds.) TID Report no. 26740

25. Kroll W J, Schlechten A W and Yerkes L A 1946 Trans. Electrochem. Soc. 89365

26. ORNL Reporter (Retiree newsletter) (November 2010) 1

27. Fischer W and Chalybaeus W 1947 The separation of inorganic mixtures by distribution between two solvents. III. The separation of hafnium from zirconium by distribution Z. Anorg. Chem. 25579

28. Miller E C 1953 Zirconium and nuclear reactors in Zirconium and zirconium alloys (Ohio: American Society of Metals) p. 327

29. Cox R P, Peterson H C and Beyer G H 1958 Separating hafnium from zirconium. Ind. Eng. Chem. 50141

30. Ref. 24 , p. 11 